6) Millán, C., Sammito, M., García-Ferrer, I., Goulas, T., Sheldrick, G.M. and Usón, I. (2015). Acta Cryst D71, 1931-1945.

Keywords: phasing, molecular replacement, small fragments, clustering, ARCIMBOLDO

\section{MS4-P7 Fast iodide-SAD phasing for membrane protein structure determination}

Igor Melnikov $^{1}$, Vitaly Polovinkin ${ }^{2,3}$, Kirill Kovalev ${ }^{4}$, Vitaly Shevchenko ${ }^{3}$, Mikhail Shevtsov ${ }^{4}$, Valentin Borshchevskiy ${ }^{4}$, Vadim Cherezov $^{5}$, Gordon Leonard ${ }^{1}$, Valentin Gordeliy ${ }^{2,3,4}$, Alexander Popov ${ }^{1}$

1. European Synchrotron Radiation Facility, F38043Grenoble, France

2. Institut de Biologie Structurale, Université Grenoble Alpes, Grenoble, France

3. Institute of Complex Systems (ICS), ICS-6, Structural Biochemistry, Research Center Jülich, Jülich, Germany

4. Laboratory for Advanced Studies of Membrane Proteins, Moscow Institute of Physics and Technology, Dolgoprudniy, Russia

5. Department of Chemistry, Bridge Institute, University of Southern California, Los Angeles, California 90089, USA

email: igor.melnikov@esrf.fr

Membrane proteins are hard to investigate. Each step of $\mathrm{X}$-ray crystallographic pipeline from protein production to structure solution is non-trivial. In particular, experimental phasing techniques have shown their ineffectiveness in case of either soaking or co-crystallization of membrane protein crystals. A fast, easy and universal method is presented here for membrane protein structure determination. Four structures of target membrane proteins from four different largest classes have been solved via single-wavelength anomalous diffraction of iodide-soaked crystals. The method is highly efficient for various data collection strategies: standard and serial crystallography at synchrotron and XFEL sources.

Keywords: SAD, crystal soaking, iodide, membrane protein 